\title{
Concepción y erotismo en la literatura médica medieval
}

\author{
$\mathrm{M}^{\mathrm{a}}$ Cruz Herrero Ingelmo \\ Universidad de Valladolid \\ mcruz@fyl.uva.es \\ Enrique Montero CARTELle \\ Universidad de Valladolid \\ enrique@fyl.uva.es
}

Recibido: 14 de septiembre de 2012

Aceptado: 29 de octubre de 2012

\begin{abstract}
RESUMEN
La creencia generalizada en la medicina antigua y medieval de la existencia de un semen masculino y otro femenino implica que, para que se produzca la concepción, el hombre y la mujer tienen que emitir el semen y simultáneamente. Como se reconoce que la naturaleza de la mujer en la eyaculación es más lenta, la puerta para el arte erótico puede abrirse mediante la indicación de los medios aconsejados por el médico para lograr la concurrencia simultánea del semen. En este trabajo se analiza en los textos médicos medievales si esta puerta se abrió o no y en qué condiciones. En tal sentido, el Canon de Avicena fue el texto clave para toda la medicina medieval.
\end{abstract}

Palabras clave: Erotismo. Concepción. Medicina medieval y renacentista.

Herrero Ingelmo, Ma . C. - Montero Cartelle, E., «Concepción y erotismo en la literatura médica medieval», Cuad. Fil. Clás. Estud. Lat. 32.2 (2012) 299-314.

\section{Conception and Eroticism in Medieval Medical Writings}

\begin{abstract}
A common belief in Ancient and Medieval Medicine is that the embryo wasn't conceived unless the male and female seeds were expelled simultaneously. As women's ejaculation was regarded to be slower than men's, medical advice about erotical techniques is welcomed in order to achieve a successful sperm union. This paper analyzes whether Medieval Medical texts dealt or not with this issue and its own particular circumstances. In this respect, Avicenna's Canon played a central role in Medieval Medicine.

Keywords: Erotism. Conception. Medieval and Renaissance Medicine.

Herrero Ingelmo, Ma ${ }^{\text {a }}$ C. - Montero Cartelle, E., «Conception and Eroticism in Medieval Medical Writings», Cuad. Fil. Clás. Estud. Lat. 32.2 (2012) 299-314.

Sumario 1. Los antecedentes hipocráticos y galénicos. 2. Relación entre medicina y erotismo. 3. La autoridad de Avicena y Razes. 4. La recepción en Bernardo de Gordon. 5. La expansión posterior. 6. Conclusiones. 7. Referencias bibliográficas.
\end{abstract}




\section{LOS ANTECEDENTES HIPOCRÁTICOS Y GALÉNICOS}

En el texto de Hipócrates, De generatione 4, se encuentran ya expresados algunos conceptos fundamentales sobre la generación, como la existencia de un esperma femenino, cuya mezcla con el masculino en el útero será causa de la generación, como cuando se afirma: «La mujer eyacula también a partir de todo el cuerpo, unas veces dentro de la matriz -y entonces se vuelve húmeda-y otras fuera, cuando la matriz es más abierta de lo conveniente... Una vez que la mujer ha realizado el coito, en el caso de que no vaya a quedar encinta, normalmente el esperma de ambos (es decir, del hombre y de la mujer) fluye fuera, pero en el caso de que vaya a concebir, el esperma no sale, sino que permanece en la matriz, etc.» (Rodríguez Blanco 2003, pp.251$253)^{1}$. En la Antigüedad grecolatina, en efecto, desde los primeros textos médicos de relieve que tenemos, como es el Corpus Hippocraticum (siglos V-IV a. C.), aparece una ginecología bastante desarrollada (Gourevitch 1966, p.2084), como se ve en las obras De morbis muliebribus, De sterilibus, De uirginum morbis, De natura muliebri, De generatione, etc.

Por el contrario, según Aristóteles, la mujer no tiene semen propiamente dicho, ya que muchas veces concibe sin haber tenido placer en el coito o bien, aunque tenga placer, no concibe, si no tiene menstruaciones, que es la materia (una especie de esperma impuro que no ha alcanzado el grado supremo de elaboración) que aporta la mujer a la concepción (De gener. anim.1.19.727b). En efecto, si expulsara semen en el coito, tendría dos secreciones espermáticas a la vez, es decir, semen y menstruación, por lo que su papel en la concepción queda reducido a proporcionar la materia que se encuentra en la sustancia de las menstruaciones ${ }^{2}$ y que es el campo en el que se desarrolla la simiente masculina (De gener. anim.1.19.727a.2530): «Puesto que esto (la menstruación) es lo que se produce en las hembras como el semen en los machos, y no es posible que se formen dos secreciones espermáticas a la vez, es evidente que la hembra no contribuye con esperma a la reproducción» (Sánchez 1994, p.107). En la biología aristotélica (siglo IV a. C.) de tanto influjo posterior ${ }^{3}$, expresada en la Historia animalium y completada por el De generatione animalium y el De partibus animalium, se niega la existencia del semen femenino y, en consecuencia, no se plantea la cuestión de si la concepción es el resultado de la mezcla de ambos por igual, como se postulaba en el Corpus Hippocraticum.

La ginecología de base hipocrática se desarrolló aún más en la escuela alejandrina (siglos IV-III) con la investigación de estudiosos de la anatomía y de la fisiología

\footnotetext{
${ }^{1}$ En el Corpus Hippocraticum se dan teorías diferentes sobre el origen del semen, pero predomina la pangénica que sostiene que procede de todas las partes del cuerpo (De generatione 3.1). Sobre los antecedentes de esta creencia y las distintas teorías sobre el origen del semen, que ahora dejamos de lado, véase Gerlach (1937-1938) y Diepgen (1937, pp.148; 168).

${ }^{2}$ Para una breve visión de este problema, $c f$. Bologne (1998, pp.46 y ss.) o Laurent (1989, pp.63 y ss.). Las posibles contradicciones en el interior de la obra de Aristóteles han sido suficientemente explicadas en Byl (1979, pp.136-152) y Manuli (1980, p.405 y n.2).

${ }^{3}$ Como, por ej., en la Edad Media latina. Cf. Martorelli (2000, pp.311-338).
} 
de diversa orientación metodológica, como es el caso del dogmático Herófilo, quien desarrolla la idea novedosa de la existencia de dos testículos productores de semen también en la mujer (es decir, los ovarios), además de los canales espermáticos (es decir, las trompas de Falopio) que lo transportan para su eyaculación, según testimonio de Galeno (De semine 2.1, Kühn 1964-1965, 4.596²):

Herophilus autem nescio quomodo effundi ait foeminarum semen, quanquam sane de testiculis exacte descripserit in tertio de dissectione ipsorum, in principio in hunc modum inquiens: "Adnati sunt in utero testiculi a lateribus ex utraque parte uteri in paucis differentes a masculis... Seminalis autem meatus in utroque non ualde apparet, adnatus est autem utero ab externa parte...»

Esta manera de ver las cosas permaneció viva en la Roma del siglo II d. C. en la concepción de la anatomía y de la fisiología femenina expresada por Sorano ${ }^{5}$, pero alcanzó gran esplendor con Galeno, que le dedica buena parte de tratados tan importantes como De usu partium, De semine, De uteri dissectione, De foetus formatione, etc. Galeno, aunque sigue a Hipócrates, lleva a cabo una síntesis propia, en la que recoge la teoría de la presencia tanto en el hombre como en la mujer de dos testículos, de función básica en la cocción del esperma (De semine 12-15, Kühn 1964-1965, 4.555 ss.), y dos tipos de semen, uno masculino y otro femenino, ambos necesarios para la concepción, en la que deben concurrir. En esta, sin embargo, la parte femenina, más fría y húmeda con relación al hombre, ocupa un lugar pasivo frente al papel creador del semen masculino ${ }^{6}$.

La ginecología medieval latina (Diepgen 1963) hereda la pugna entre la biología aristotélica y la ginecología hipocrático-galénica acerca de la existencia del semen masculino y femenino y del papel de la mujer en la generación, tratando en muchos ocasiones de conciliar, mediante hábiles recursos escolásticos, ambas posiciones encontradas ${ }^{7}$. Podemos decir, simplificando la situación, que los médicos siguieron la tradición hipocrático-galénica, mientras que los teólogos prefirieron la aristotélica ${ }^{8}$.

\footnotetext{
${ }^{4}$ Citamos para comodidad del lector por la versión latina de Kühn (1821-1833, reimpr. 1964-1965). Estas ideas se encuentran desarrolladas en Staden (1989, pp.165-169).

${ }^{5}$ Quien en su Gynaecia ( $c f$. Burguière-Gourevitch-Salinas 2003, 1.3.1-5), desde la perspectiva de la escuela metódica, cuya adaptación latina por Celio Aureliano y Musción le proporcionó gran difusión, rechaza la distinción cálido-frío o húmedo-seco y, por lo tanto, la fisiología de corte hipocrático, para tratar la patología de la mujer como la del hombre de modo semejante, salvo lo que por naturaleza es propio de ella y que es el objeto de su obra.

${ }^{6}$ Para estas cuestiones véase Montero-Conde (2001, pp.373-399).

${ }^{7}$ Una visión general de esta problemática se puede ver en Bologne (1998, pp.45 y ss.). La conciliación de ambas posturas en el caso concreto de Mondino la ha estudiado Martorelli (2000, pp.335 y ss.). También existe una buena síntesis sobre la problemática de la mujer en la Roma antigua en el estudio de Gourevitch (1984).

${ }^{8}$ El contexto general sobre la sexualidad en la Edad Media en el que se inserta este trabajo puede verse en estudios como los de Cadden (1993); Mazo Karras (2005); Harper - Proctor (2008), etc.
} 


\section{RELACIÓN ENTRE MEDICINA Y EROTISMO}

En esta perspectiva, el posible punto de unión entre medicina y erotismo lo plantea la idea fundamental de la necesidad de la presencia simultánea del semen masculino y del femenino como requisito imprescindible para que exista la concepción. Independientemnte de posibles objeciones, resueltas de mucha formas, como la concepción en mujeres frígidas o violadas, el hecho es que para la concepción es necesario que hombre y mujer emitan el semen y al mismo tiempo. Como se reconoce que la naturaleza de la mujer en la eyaculación es más lenta, la puerta para el arte erótico puede abrirse mediante la indicación de los medios aconsejados por el médico para lograr la concurrencia simultánea del semen masculino y el femenino.

En la medicina grecolatina parece que esta puerta no se abrió. No se dan indicaciones especiales ni sistemáticas en este sentido.

Hipócrates, en efecto, reconoce la necesidad de la emisión simultánea de los dos espermas, por ejemplo, en De morbis muliebribus 1.17 (Littré 1839-1863, 7.56) cuando señala que «si el semen del hombre confluye directamente con el de la mujer, esta concebirá» (Sanz 1998, p.79), pero se despreocupa del modo de lograrlo, a pesar de que reconoce el papel del placer y del orgasmo en la eyaculación de la mujer en De generatione IV y V y se preocupa de los obstáculos que impiden la concepción en su tratado De sterilibus.

A Aristóteles este problema no le preocupaba por su teoría de la inexistencia del semen femenino e incluso en un relevante capítulo del De generatione animalium (1.19.727b) rebate la teoría del Corpus Hippocraticum, como hemos indicado. Por ello resalta la contradicción que supone la afirmación de Historia animalium 10.636b1-25 de la necesidad de que hombre y mujer eyaculen simultáneamente para la concepción: «En efecto, si la mujer proporciona su parte de esperma y contribuye a la generación, es evidente que los esposos han de ir al unísono» (Pallí 1992, pp.578-579). Tal contradicción ha llevado a pensar en el carácter espurio de este libro, extremo innecesario, pues basta explicar de otro modo el sentido que Aristóteles da aquí al término sperma como humor no seminal, sino uterino (Byl 1979, pp.136-152; Manuli 1980, p.405 y n.2). En todo caso ello tampoco da pie para explicaciones del modo como lograr la emisión seminal simultánea.

Situación similar nos presenta Galeno dentro de su manera de ver las cosas, como hemos indicado. Para él el semen femenino, aunque sea imperfecto, por su condición fría y húmeda (De usu partium, 14.6, Kühn 1964-1965, 4.164), es necesario para la concepción, por lo que la mujer tiene que estar excitada y sentir placer (De usu partium, 14.11, Kühn 1964-1965, 4.188-189) para lograr la emisión seminal al mismo tiempo (De semine 1.7 y 2.1, Kühn 1964-1965, 4.536.601). Con todo también Galeno pasa por alto la técnica para lograrlo.

\section{LA AUTORIDAD DE AVICENA Y RAZES}

En el mundo árabe, las ideas griegas sobre la generación, particularizadas en Galeno y Aristóteles, fueron bien conocidas y asimiladas (Schipperges 1964, pp.330$35 ; 55-84 ; 89-91)$. Al mundo latino medieval llegaron, tras la pérdida del conocimiento 
de las fuentes griegas, a través de las traducciones de los grandes médicos árabes, de las que destacamos el Pantegni de 'Ali ibn Al-'Abbas (siglo X), traducido a finales del siglo XI por Constantino el Africano. Fue la obra de mayor influjo en la medicina medieval (Montero 1990, pp.65-88) hasta la traducción del Canon de Avicena (siglos X-XI) en Toledo de Gerardo de Cremona en la segunda mitad del siglo XII, pero de difusión más tardía a lo largo del siglo XIII. La importancia del Canon como fuente, que permaneció como autoridad casi indiscutible hasta el Renacimiento y discutida, pero viva, dentro de él, indica la amplitud que estas ideas alcanzaron en los siglos siguientes (Siraisi 1987).

Pero en el corpus árabe traducido por Constantino no se entra en consideraciones sobre el modo de lograr la emisión seminal al unísono, como se ve en el Viaticum, ni tampoco en el Pantegni, ni incluso, a pesar de su título, en el Liber de coitu, que es una traducción llevada a cabo por Constantino de una obra homónima de Ibn al-Gazzar (Montero 1988, pp.213-223), uno de los célebres médicos del período islámico tunecino (siglo X), discípulo de Isaac Judaeus (autor también del citado Viaticum traducido por Constantino el Africano). Estas obras y en concreto el Liber de coitu se interesan por la fisiología, la patología y la terapia del coito y no por la generación, por lo que no entran en la perspectiva de los recursos para la generación que nosotros buscamos. Con todo, en el cap. II 16, dedicado a los medicamentos para favorecer la libido -y siempre en una perspectiva masculina-, señala de pasada que hay otro tipo de recursos para aumentar el apetito sexual atribuidos a la autoridad de Filúmeno:

Ait Filumenus libidinem diuersis modis fieri, quia quicquid ei adiunctum est accendit eam, ut amor apparens et recordatio amoris et oscula maxillarum, constriccio manuum et cum sugitur lingua et contemplacio uultus dilecte et suspiratio murmurosa. Hec et his simila adaugent libidinem.

Dejando de lado la dudosa paternidad de Filúmeno (siglo II d. C.), pues, si no se trata de una deformación de un nombre árabe, este pasaje es desconocido en la obra conservada ${ }^{9}$, es evidente que se trata de procedimientos eróticos para excitar la libido, interesantes por lo que suponen de novedad en esta época en el mundo medieval, pero que no están orientados directamente a lograr la eyaculación unísona, entre otras razones por la orientación masculina del tratado.

Tenemos que llegar a la traducción del Canon de Avicena para encontrarnos con el primer texto en la medicina latina medieval que trata directamente de los medios para lograr la eyaculación simultánea de los dos espermas, porque Avicena devuelve a Occidente las ideas hipocrático-galénicas pasadas por el tamiz de la cultura árabe. En efecto, en Can.3.Fen.20 y 21 (Avicena 1527) recoge la idea de los dos semina y señala las condiciones óptimas para la concepción, una de las cuales es la emisión simultánea del hombre y de la mujer. Como hay distintas complexiones y la mujer puede tener diversas maneras de ser en cuanto a la eyaculación, aconseja Avicena sobre cómo el hombre

\footnotetext{
${ }^{9}$ Los fragmentos conservados se encuentran interpolados en el libro II de la Therapeutica de Alejandro de Tralles (siglo VI d. C.). Sobre estos problemas $c f$. Montero (1983, p.161 y notas).
} 
debe conducir el «juego» sexual para lograr la emisión simultánea. Así en Fen.21. trat.1.10.f.288v., expone, hablando de la esterilidad, como preparación remota:

oportet ut eligatur conuenientior horarum coitus et iam diximus eam. Et de eis eligatur conuenientior, ut sit in fine menstrue et in hora simili hore in qua oportet ut in ea coitus fiat, propter illud quod diximus. Et oportet ut prolongetur dimissio coitus tanta prolongatione, ne proueniat ut corrumpat ea ambo spermata frigus... Deinde dimittant ipsum tamdiu quod sciatur quod sperma bonum iam aggregatum sit et consideretur de eis ut sit illud in prima hora mundificationis eius.

A continuación viene la parte referida a la realización del coito:

Amplius prolongetur ludum et proprie cum mulieribus quarum complexiones non sunt male. Tangat ergo uir eius mamillas cum facilitate et tangat pectinem eius et obuiet ei non permiscendo se ei permixtione uera. Qumque desiderat et affectat, permisceatur ei fricando de ea quod est inter anum eius desuper et uuluam. Ille enim locus est locus delectationis eius. Consideret ergo in ea horam in qua fortis fit in ipsa adherentia et incipiunt oculi eius mutari in rubedinem et eius anhelitus eleuari et uerba eius balbutire et tunc mittat illic sperma oppositum ori matrici, dilatando locum eius illic parumper, scilicet, tanta quantitate, ne consequatur ipsum impressio aeris exterioris omnino. Ipse enim in dispositione corrumpit ipsum et non est conueniens ad generationem.

Después acaba la exposición con varias advertencias finales para facilitar la concepción:

Et scias quod quando mittitur sperma in hora sua parum..., tunc perditur sperma; immo oportet ut recipiat os matricis cum eiectione quadam et non stringat in uirga exitum, immo adhereat ei hora, qum iam permiscetur post illud permixtione que est uehementioris perscrutationis, donec uideat quod nodi oris matricis et anhelitus eius iam quiescunt ex toto; et post illud < expectet> parum ipsa iacente eleuatis coxis et demerso dorso. Deinde surgat ab ea et dimittat eam sic horula una cum strictis pedibus et retento anhelitu. Et si dormierit post illud, erit melius ad conceptionem.

A la advertencia final de Avicena sobre el cuidado que se debe tener para evitar la entrada del aire en el momento de la emisión seminal, porque, si ello ocurre, se produce la corrupción del semen, añade Avicena con gran énfasis, como prescripción fundamental, que de no producirse la emisión seminal en el momento oportuno (es decir, al unísono hombre y mujer) no es posible la concepción. Una vez lograda la conjunción de espermas, a continuación expone Avicena otros consejos sobre la posición e inmovilidad de la mujer para retener el semen y lograr la concepción, entrando ya en otros aspectos del engendramiento.

Dejando de lado la preparación remota y otras cuestiones generales, los elementos que se enumeran como ludus o praelusio coitus en la emisión seminal son los siguientes:

1. tangat ergo uir eius mamillas: tocar los pechos.

2. tangat pectinem: tocar el pubis. 
3. obuiet ei non permiscendo se ei permixtione uera: contacto físico corporal, simulando la postura del coito.

4. fricando de ea quod est inter anum eius desuper et uuluam: friccionar el perineo.

5. permisceatur ei: realizar la penetración.

Después se señala como momento oportuno de la emisión seminal (tunc mittat illic sperma), cuando se observan las siguientes condiciones en la mujer, señal de que se aproxima su «emisión» seminal:

1. fortis fit in ipsa adherentia: presión contra el varón.

2. incipiunt oculi eius mutari in rubedinem: ojos enrojecidos.

3. eius anhelitus eleuari: jadeos.

4. uerba eius balbutire: balbuceos.

He aquí, pues, un texto clave que podría haber abierto la puerta entre medicina y erotismo en el mundo medieval. No obstante, Avicena se sitúa tanto en esta como en todas las demás recomendaciones que da sobre las relaciones sexuales (como, por ejemplo, Can.3.Fen.20.tract.1.11, en donde se detiene incluso en las posiciones del coito útiles o nocivas para la concepción) en una perspectiva estrictamente médica y neutra, que marcó la pauta para todos los demás autores que trataron del tema. Las revelaciones sobre las zonas erógenas de la mujer, de los mecanismos para excitarla y de los signos reconocibles de la proximidad de la eyaculación se mencionan fríamente, como si de una operación quirúrgica se tratase. En efecto, si se lee con atención el texto, se observa que el hombre no busca su placer directamente, sino el de la mujer y, si busca el placer de la mujer, no es por sí mismo, sino para lograr la emisión seminal simultánea oportuna para la concepción. La técnica sexual que se describe es solo un medio para un fin superior, por lo que no parece oportuno hablar de erotismo ${ }^{10}$.

Esta descripción de Avicena, por sencilla que sea, se inserta en una rica tradición árabe, heredera y asimiladora de diferentes culturas, como la India, de la que Avicena es su mayor exponente. En efecto, ya hemos señalado la vertiente erótica del $\mathrm{Li}$ ber de Coitu de Ibn al-Gazzar, traducido por Constantino. Tenemos también, entre otros, el De coitu de Maimónides (siglo XII) traducido por Juan de Capua (finales del siglo XIII y comienzos del XIV) (Burnett 2011) y, ya más tarde, en la Granada nazarita del siglo XIV, el Libro del cuidado de la salud durante las estaciones del año o Libro de Higiene de Ibn al-Hatib (Vázquez de Benito 1984, pp.149-159.) ${ }^{11}$, que res-

\footnotetext{
${ }^{10}$ Por lo demás, no se trata de un erotismo con el matiz de exceso que se suele dar a este término: $C f$., por ejemplo, Sillamy (1962, p.115), o bien Pellegrini (1968, p.829). Por eso parece apropiada la expresión «sexualité medicaliseé» de Jacquart-Thomasset (1985, p.181), al referirse a este tipo de prescripciones, aunque se encuentra en un capítulo que titulan «L'érotisme retrouvé».

11 También en catalán el Speculum al foderi (Solomon 1990) es un texto de finalidad erótica que, en nuestra opinión (Cf. Montero 1991, pp.71-80), añade al anónimo salernitano Liber minor de coitu una fuente árabe que incorpora la técnica amatoria árabe e india.
} 
ponden a una tradición de cultivo de la higiene sexual en la que la medicina incorpora una serie de elementos eróticos para la satisfacción de la pareja, tengan a la vista o no la generación ${ }^{12}$.

Pero disponemos también del testimonio de Razes (Ar-Razi) (siglo IX-X). Su manual de conjunto, conocido en occidente como Liber de medicina ad Almansorem (Razes 1497, trat. 5.72. f.27ra), traducido al latín en la segunda mitad del siglo XII por Gerardo de Cremona, tuvo una gran difusión. Este autor, que trata numerosos aspectos de la ginecología, también expone brevemente del modo de realizar el coito para lograr la concepción:

De hiis que ualent ad impregnadum.

Ad hoc ualet ut uir et mulier non coeant nisi post longum tempus et ut non sint ebrii et ut uir cum muliere ludat tamdiu donec eam uideat desiderare coitum, quod in oculis mulieris atque anhelitu cognosci potest. Et sit coitus post menstruorum purgationem. Qui postquam cum ea coire ceperit, ipsius uehementer eleuet crura, et sit tunc mulieris caput inferius mersum atque uir a spermatis emissione abstineat, donec sciat mulierem suum impleuisse desiderium. Et tunc ipse semen effundat. Mulier uero diu iaceat...

En cuanto al contenido, esta exposición muestra una coincidencia general con Avicena, aunque el tratamiento de Avicena es mucho más elaborado y extenso ${ }^{13}$.

\section{LA RECEPCIÓN EN BERNARDO DE GORDON}

La primera documentación que hemos advertido del influjo de estas ideas es el testimonio del profesor de Bolonia Guillermo de Saliceto, quien a finales del siglo XIII compuso una Summa conseruationis et curationis. Dentro del capítulo De his que ualent ad impregnandum (Saliceto 1490, fol.66va-66vb.), en la parte referida a la preparación remota señala que uir et mulier abstineant longo tempore coitu..., que conueniens ad intentionem istam est ut uir neque mulier sint ebrii y que utile uidetur ut hora coitus sit inmediate post menstruorum purgationem. Estos datos y su formulación lingüística derivan directamente de Razes.

Sin embargo, en la exposición siguiente sobre el modo de hacer el coito, no sólo sigue a Avicena, sino que lo cita directamente como su fuente:

Tunc enim uir incipiet ludere cum muliere tangendo mamillas et pectinem et fricando marginem siue maceraim, id est, loca pilosa qua sunt circa uuluam, nam medici uolunt quod iste sit locus fortissime delectationis in muliere et hoc narrat Auicenna libro III in regimine et curatione sterilitatis,

\footnotetext{
${ }^{12}$ Sobre este tipo de literatura véase Sarton (1927-1948, 2.1. p.79 ss.); Montero (1983, pp.31-38); JacquartThomasset (1985, pp.170 y ss.); Arjona (1992).

${ }^{13}$ El único punto que podría desentonar con el texto de Avicena, es decir, la ebriedad como impedimento de la concepción, lo menciona también Avicena antes (Can.3.Fen.20.tract.1.8.f.287vb) en el capítulo titulado De sterilitate et difficultate impregnationis, cuando dice: Et de genere quidem spermatis ex quo non fit generatio est sperma infantis et patientis nauseativam satietatem et ebrii et senis.
} 
con la salvedad de que desconoce el nombre de lo que expresa Avicena, mediante una perífrasis, como ea quod est inter anum eius desuper et uuluam, es decir, el perineon, y recurre a una denominación maceriam que carece de paralelo.

A pesar de ello, en la exposición siguiente vuelve a depender de Razes, a juzgar por expresiones como hora coitus mulieris crura uehementius eleuet y sit hora illa caput mulieris inferius mersum. Abstineat uir etiam hora coitus ab emissione sui spermatis, donec nouerit mulierem suum impleuisse desiderium. Por último, antes de añadir la frase siguiente de Razes et suum emittat semen, añade de su propio cosecha la precisión: et tunc uir trahat naticas mulieris ad se et uiolenter firmet se cum genitalibus uersus mulierem. Sin embargo, Razes no es citado, lo que redunda en beneficio de la fuente por excelencia que era Avicena.

Pero el texto de Avicena influyó en Bernardo de Gordon, uno de los maestros de Montpellier, más famosos de la Edad Media, quien en 1305 termina su obra más conocida, el Lilium medicinae ${ }^{14}$. Esta obra, dentro de un cierto nivel científico, es un manual muy logrado con fines didácticos y de vulgarización, que alcanzó en la Edad Media una gran difusión, a juzgar por la gran cantidad de manuscritos que lo transmiten, las ediciones que lo divulgaron y su temprana traducción a diversas lenguas (Demaitre 1980, p.51). Bernardo de Gordon, en su Particula VII, en la que estudia las enfermedades de los miembros de la generación, dedica el capítulo 14 (Gordon 1542) a la esterilidad de la mujer, en el que no olvida una sustanciosa exposición sobre el Modus coeundi (f.334r-v) oportuno para la concepción, como hacía Avicena:

Vir et mulier debent esse temperati, uergere ad temperamentum et debent esse ordinati in cibis, potibus et aliis sex rebus non naturalibus, cibo digesto et superfluitatibus expulsis, post mediam noctem et ante noctem masculus debet excitare foeminam ad coitum loquendo, osculando, amplexando, mammillas tangendo, pectinem et peritoneum, et hoc totum fit ut mulier appettat ut semina concurrant simul, cum mulieres tardius emittant, et cum mulier incipit loqui quasi balbutiendo, tunc debent se commiscere et paulatim debent coire et debet se unire omnino pectini mulieris ne aer possit ingredi, et post seminis effusionem debet stare supra mulierem sine motu; non enim statim debet surgere et postquam surrexerit debet extendere crura, stet resupina et dormiat, si potest, quia multum est utile...

Como se observará, las prescripciones responden sustancialmente a los consejos de Avicena con variaciones sólo de detalle, pues Bernardo de Gordon, que se detiene un poco más en los preliminares mediante la conversación, los besos (que ya estaba en Constantino, De coitu) y los abrazos, denomina directamente la zona erógena como peritoneum evitando la perífrasis de Avicena (aunque confundiendo perineum con peritoneum ${ }^{15}$, error que no sabemos si se debe a la transmisón textual o con menor probabilidad al propio autor, que emplea en otras ocasiones este término en su acepción propia, como en 7.1.315v16) y, por último, al considerar como señal inequívoca de

\footnotetext{
${ }^{14}$ El libro de Demaitre (1980) ofrece un estudio de conjunto muy completo sobre este autor. La versión española de esta obra puede verse en Cull-Dutton (1991) y en Dutton-Sánchez (1993).

${ }^{15}$ En los textos médicos medievales se documentan las formas perineon, perineos y perin(a)eus.
} 
la llegada del orgasmo balbutire, ya no estima necesario indicar también la elevación de la respiración y la rojez de los ojos ${ }^{16}$.

Este excursus de Bernardo de Gordon tuvo una gran difusión en la literatura ginecológica por una razón: la Particula VII del Lilium de Bernardo de Gordon fue la fuente principal de las monografías sobre la esterilidad que, inaugurando el género, aparecen en el siglo XIV dentro del ámbito de influencia de la esplendorosa escuela médica de Montpellier. Son un conjunto de tratados anónimos, por más que figuren atribuidos a los nombres más sonoros de esta escuela, de autores de segunda fila y de carácter eminentemente práctico (por lo que no se interesan en cuestiones teóricas). El tratamiento de la esterilidad es en ellos bastante tópico y repetitivo debido a que su fuente principal es precisamente el Lilium de Bernardo de Gordon (Montero 1999). Por ello, podemos decir que a partir de entonces la breve exposición de Razes perdió su influencia.

Así, en el anónimo originario de Montpellier Tractatus de sterilitate (Montero 1993) del siglo XIV, entre las muchas recomendaciones de carácter práctico contenidas en el cap. II, 9 dedicadas al Modus operandi contra sterilitatem, añade las siguientes en las líneas 124-129:

Vir autem, quia mulieres sunt uerecunde, cum durmierit primum sompnum, debet mulierem palpando mamillas et uentrem excitare et ad coytum prouocare, quam cum uiderit bene accesam et calefactam, tunc erit tempus accedendi ad eam, quia tunc ambo semina concurrunt eodem tempore, quod est de necessitate concepcionis.

En este texto no está detrás Avicena, sino Bernardo de Gordon simplificado, como indica el momento elegido, el sistema de excitación y los signos del momento del orgasmo femenino, aspecto que se sintetiza ahora con la expresión mulier accensa et calefacta. La expresión calefacta no la hemos documentado antes en ningún texto en este sentido.

Probablemente este uso debía encontrarse en circulación en el ámbito de influencia de Montpellier, ya que, aunque no se encuentra en la fuente, que es Bernardo de Gordon, lo vemos reiterado en otra de las monografías dedicadas a la esterilidad del mismo ámbito de las que comentamos y que es también del siglo XIV. En efecto, en el cap. X, líneas 15-20, del Tractatus de conceptu (Conde-Montero- Herrero 1999, pp.86-87) se explica de nuevo la técnica para lograr la emisión seminal conjunta de la siguiente manera:

Post dormiat donec de mane in aurora, expulsis superfluitatibus, iungant se donec sint ambo per ludum tangendo mamillas et peritoneum calefacti; et uir abstineat a coytu per aliquod tempus et faciat quod ambo spermata simul concurrant et sint iuncti taliter quod uentus non subintret et appodiet se uir fortiter supra pectus mulieris et stet ibi donec mulier resumpserit anhelitum.

En el texto vuelve a imperar el magisterio de Bernardo de Gordon, con el que coincide en la expresión tangere mamillas (frente a palpare del Tractatus de sterilitate),

${ }^{16}$ Sobre la posición de Bernardo de Gordon en este aspecto $c f$. Thomasset (1992, p.363). 
se copia el error de la designación del peritoneum por perineum (frente a uentrem) y se insiste, además de la importancia de la emisión conjunta, en la técnica para que no entre aire que corrompa el semen, que el Tractatus de sterilitate se había pasado por alto. Desde el punto de vista lingüístico reaparece el término calefacti, esta vez aplicado al hombre y a la mujer, lo que para nosotros es indicio evidente de la influencia de Montpellier.

En consecuencia, el magisterio de Avicena a través de la obra más popular de Bernardo de Gordon adquirió una notable difusión, hasta el punto de aparecer en dos de los tratados especializados sobre la ginecología y, en concreto, sobre la esterilidad de carácter práctico que circularon en la Edad Media tardía. Esto nos hace suponer que la difusión de la exposición de Avicena sobre este punto tuvo que ser mayor.

Un ejemplo más nos da pie para ello. En efecto, en la Rosa Anglica de Iohannes Gaddesden (1492), quien a comienzos del siglo XIV, unos años después de la conclusión del Lilium de Bernardo de Gordon, escribe una especie de summa o compendium medicinal, los problemas de la concepción reciben la atención debida. Este autor recobra la exposición de mecanismos para lograr la emisión seminal conjunta, abriendo la puerta a una exposición más amplia, pero que evidencia el conocimiento directo no de Avicena, sino del texto de Bernardo de Gordon del que está tomado:

ante diem debet masculus excitare feminam ad coitum loquendo, osculando, amplexando mammillas, tangendo pectinem et peritoneom et totam uuluam in manu accipere et super nates percutere, et hoc totum est ut mulier appetat et semina concurrant, quia ut plurimum mulieres tarde emmittunt; et cum mulier incipit loqui balbutiendo, tunc debent se coniungere seu commiscere et paulatim debent coire; et oportet se unire omnino pectori mulieris ne aer possit ingredi, similiter pectini quantum potest; et post seminis emissionem debet stare et iacere super mulierem sine motu et non statim surgere; et postquam surrexerit debet mulier extendere crura et iacere resupina et dormire ne semen exeat...

En este texto, salvo el procedimiento totamque uuluam accipere in manus et super nates percutere que, al no encontrarse en las fuentes, consideramos de su propia cosecha, se sigue el tenor del texto de Bernardo de Gordon, incluso en el error peritoneum por perineum, salvo la sustitución de algún término por sus sinónimos. Con todo, a continuación añade Iohannes Gaddesden unos consejos de técnica sexual sobre situaciones no mencionadas en las fuentes vistas, como el caso de que la mujer no haya eyaculado en el coito (por lo que no ha concebido) y sea necesario un segundo coito a la mañana siguiente, terreno en el que el discurso se aleja de Avicena y Bernardo de Gordon:

Item si uirga erigatur tarde post primum coitum et uis $2^{\circ}$ coire in mane, ut forte mulier quae non emissit sperma compleat desiderium suum; et hoc potest scire, si ipsa post coitum bene post approximetur et osculetur et amplexetur et post ponat manum quasi de abrupto ad uirgam et testiculos; et hoc facit ut uideat si tu sis paratus iterato ad coitum. Tunc enim bonum est $2^{\circ}$ coire. 


\section{LA EXPANSIÓN POSTERIOR}

En adelante, y dentro siempre de esta perspectiva masculina, Avicena seguirá siendo la fuente básica para los preceptos médico-eróticos de la emisión seminal conjunta, aunque el magisterio de Bernardo de Gordon no ha quedado en el olvido. Valgan, como ejemplo, tres testimonios de mediados del siglo XV.

El médico paduano Miguel Savonarola en su afamada Practica, entre sus prescripciones utilia generationi, tras los tópicos de la preparación remota, vuelve a la misma exposición que hemos visto, a la que añade la precisión, dentro de tangere mamillas, el lugar preciso que acariciar para excitar a la mujer (specialiter capita mamillarum oscula iungere):

debet uir mulierem tangere ut circa mamillas et leuiter et specialiter capita mamillarum oscula iungere, et uerba prouocantia dicere, et cum uirga uulue appropinquando non intromittendo, et ultimo tangendo et aliqualiter fricando locum medium inter uuluam et anum et est locus maxime delectationis. Deinde uirgam intromittere et aliqualiter prolongare ludum ut super hoc expectet ut emissio spermatis mulieris quod oculi eius rubeant et anhelitus eleuetur et quod lingua balbutiet. Item cum uirga est intus, mulier stringat coxas et uir non se moueat ducendo anum sursum et deorsum, ne aer ingrediatur corrumpens sperma. Stet itaque sic fixus emittendo et cum sentit se emittere sperma, emittat ipsum totum... (Savonarola 1502, 6.21.ff.251va).

Por otro lado, en la preceptiva del profesor de Pavía, Antonio Guainerio, cuya obra Commentariolus de aegritudinibus matricis muestra gran experiencia clínica práctica (Lemay, Rodnite H. 1985, pp.317-336), se advierte, junto al influjo básico de Avicena y secundario de Bernardo de Gordon, algunos elementos peculiares, como son los procedimientos afrodisíacos que recomienda al final e incorpora la precisión mammillarum papillas digitis leuiter pertractet que encontrábamos en Savonarola:

Antequam igitur ad coitum uir accedat, in mulieribus amplexibus familiariter se ponat et cum suaui labiorum succione oscula det plurima, uerba dulcia et amorem inducentia proferat, mammillarum papillas digitis leuiter pertractet, et, quia in actu tali omnia facere fas est, locum inter anum et uuluam leuiter confricet. Ea enim fricatio, ut autores ferunt plurimi, in muliere delectationem affert maximam. Sepe ut uenereum actum perficiat operam det, donec tamen mulieris oculos scintillare percipiat eum interrumpet semper. Cumque mulieris scintillant oculi ac uerba truncata loquitur sic quod in medio suorum uerborum lassata sistit et in motibus suis modum habet nullum, tunc uir statim piper aut cebebas masticet et cum saliua ex tali masticatione causata uirgam illiniat uel idem cum felle capre et puluere seminis urtice faciat uel puluerem satirionis in modum ungenti cum melle conficiat cum quo tunc uirgam inungat...,

con todo lo cual se consigue el fin deseado, es decir, ut in eodem instanti spermata simul emittant (Guainerio 1497).

Por último, Bartolomé Montagnana, colega de Miguel Savonarola en Padua, en el Consilium CCVI titulado De sterilitate (1497, ff.237vb-238va) en el que señala 16 regulae et instrumenta obseruanda como Decimunquintum instrumentum includens actus 
necessarios ad fecundationem, tras remitir a las habituales consideraciones generales, da las siguientes recomendaciones que deben guardar tanto la mujer como el varón:

mulier qui fuit sterilis quando intendit se commiscere uiro... non multum festinanter copuletur illi. Et audiat etiam uir capitulum istud. Ita quod parum, uidelicet, per unam horam aut circa ante copulationem ludat uir cum muliere et econuerso cum osculis et tactu mamillarum et suminis et partium genitalium, tempus hoc expendendo quousque uir uiderit mulierem discoloratam, factam rubicundam et anhelitum parumper frequentatum et leue motum saltuosum circa partes inguinales et suminis perceptum sensu tactus. Tunc ergo uir copulet se mulieri cruribus ad alta notabiliter eleuatis, et cum senserit anhelitum parumper frequentatus magis quam prius, tunc effuso in ipsa semine, parumper stet ei copulatus, et recedens ab ea faciat ipsam reuolui super dextro latere...

Siguiendo la línea que estamos analizando, Bartolomé Montagnana introduce la nota de la ansiedad de la mujer por tener hijos (mulier qui fuit sterilis quando intendit se commiscere uiro... non multum festinanter copuletur illi) que puede dificultar la concepción, la implicación del hombre en esta tarea (Et audiat etiam uir capitulum istud), cerrando de nuevo el círculo de la tradición, con una redacción distinta, que remonta a Avicena sobre una técnica que podemos considerar ya asumida ${ }^{17}$.

\section{CONCLUSIONES}

En el tratamiento médico de la concepción, los médicos se ven obligados a expresar los medios para lograr la concurrencia simultánea del semen, sin la cual no hay concepción. Para ello acudieron al magisterio de Avicena, directamente o bien a través de B. de Gordon, con olvido de la exposición de Razes. Pero no fueron más allá, salvo detalles, del tratamiento de las fuentes y siempre dentro del capítulo dedicado a favorecer la concepción o a evitar la esterilidad. En ningún momento los consejos médicos tratan el tema como la búsqueda del placer por sí mismo, sino como medio para lograr la concepción (que, por lo demás, era su campo y, por ello, no se sintieron atrapados por cuestiones morales o de pudor. De hecho solamente Miguel Savonarola inicia su exposición en este punto con la advertencia: non dubitabo scribere que sunt utilia generationi, etsi non uideantur auditui honesta). La puerta entre medicina y erotismo, en realidad, nunca se abrió en la medicina medieval, aunque los autores van introduciendo nuevas aportaciones, probablemente propias, en las que el fin de la concepción va quedando cada vez más lejos y la técnica sexual adquiriendo mayor importancia, como ocurre, por ejemplo, en la técnica de los modos o figuras del coito ${ }^{18}$. Por el con-

\footnotetext{
${ }^{17}$ Como ejemplo de la pervivencia de estas ideas, véase también, ya a finales del siglo XVI en Paré (1586, p.408), el cap. titulado Quae in coitu ad generationem observanda sint del famoso cirujano francés Ambrosi Paraei... de hominis generatione liber.

${ }^{18}$ En su tratamiento se podría hacer un recorrido muy similar al que aquí hemos llevado a cabo en este trabajo, pues al fin y al cabo es un ingrediente más en la consecución de la concepción. Sobre esta temática véase Montero (1991b, pp.129-138); Gourevitch (1966, pp.2102-2103); Jacquart - Thomasset (1985, pp.185-187). 
trario, en el mundo literario medieval la autoridad para el arte de la realización del acto sexual fue Ovidio (Montero 1987, pp.25-26), en los versos 703-733 del libro II del Arte de amar, en una perspectiva por supuesto muy diferente: la consecución del placer de la pareja (Montero 2008, pp.97-116).

Pero en todo caso, tanto en el arte de amar de origen oriental importado por la traducción de Avicena y difundido por Bernardo de Gordon, como en el occidental ovidiano, la perspectiva, de acuerdo con los cánones de la época, es siempre masculina: son indicaciones de actuación para el marido, para el varón, y el placer femenino es un instrumento sólo para la concepción o para la satisfacción del hombre.

\section{REFERENCIAS BIBLIOGRÁFICAS}

ArJona, A. (1992), La sexualidad en la España musulmana, Universidad de Córdoba, Córdoba. Avicena, (1527), Auicenne Liber canonis medicinae, cum castigationibus Andree Bellunensis... translatus a magistro Gerardo Cremonensi..., Venetiis, in aedibus Luce Antonii Junta florentini.

Bologne, J.C. (1998), La naissance interdite, Stérilité, auortement, contraception au Moyen Age, París, Orban.

Burguière, P.- Gourevitch, D.- Salinas, Y. (eds.) (2003), Soranos d'Ephèse, Maladies des femmes, I, París, Les Belles Lettres.

Burnett, CH. (2011), «The Latin Version of Maimonides 'On Sexual Intersourse' (De coitu)», en Glaze, F.E.-Nance B.K. (eds.), Between Text and Patient, Florencia, Sismel-Ed. del Galluzo, pp.467-480.

BYL, S. (1979), Recherches sur les grandes traités biologiques d'Aristote, Bruselas, pp.136-152.

Cadden, J. (1993), Meaning of Sex Difference in the Middel Ages. Medicine, Science and Culture, Cambridge, Cambridge University Press.

Conde, P.P.- Montero Cartelle, E.- Herrero Ingelmo, M.C. (eds.) (1999), Tractatus de conceptu. Tractatus de sterilitate mulierum, Valladolid, Universidad de Valladolid.

Cull, J.- Dutton, B. (1991), Bernardo de Gordon. Lilio de medicina, Madison, University of Wisconsin, Hispanic Seminary of Medieval Studies.

Demaitre, L.E. (1980), Doctor Bernard de Gordon: Professor and Practitioner, Toronto, Pontifical Institute of Medieval Studies.

DiePGEN, P. (1937), Die Frauenheilkunde der alten Welt, Handbuch der Gynäkologie, Munich.

Diepgen, P. (1963), Frau und Frauenheilkunde in der Kultur des Mittelalters, Stuttgart, G. Thieme Verlag.

Dutton, B.-SÁnchez, M.N. (1993), Bernardo de Gordon, Lilio de medicina, Madrid, Arco Libros.

Gaddesden, J. (1492), Rosa Anglica, Papiae, Joannes Antonius Biretta.

Gerlach, W. (1937-1938), «Das Problem des 'weiblichen Samens' in der antiken und mittelalterlichen Medizin», Sudhoffs Archiv 30, 177-193.

Gordon, B. DE, (1542), Bernardi Gordonii omnium aegritudinum a uertice ad calcem opus... quod Lilium Medicinae appellatur, Parisiis, Dionysius lanotius.

Gourevitch, D. (1966), «La gynécologie et l'obstétrique», en Aufstieg und Niedergang der römischen Welt, Berlin-New York, De Gruyter, 2.37.3, pp.2083-2146. 
Gourevitch, D. (1984), Le mal d'être femme, París, Les Belles Lettres.

Guainerio, A. (1497), Commentariolus de aegritudinibus matricis editus per... Antonium Guaynerium... en Practica Antonii Guainerii Papiensis..., Venetiis, per Bonetum Locatellum. Harper, A.- Proctor, C. (eds.) (2008), Medieval Sexuality. A Casebook, Nueva York, Routledge. JaCQuart, D.- Thomasset, C. (1985), Sexualité et savoir médical au Moyen Age, París, PUF. KüHN, C.G. (ed.) (1821-1833), Cl. Galeni Opera omnia, Leipzig, Cnobloch (reimpr. Hildesheim, Olms, 1964-1965).

Laurent, S. (1989), Naître au Moyen Âge. De la conception à la naissance: la grossesse et l'accouchement (XII-XV siècle), Paris, Le Léopard d'Or.

LEMAY, Rodnite H. (1981), «William of Saliceto on Human Sexuality», Viator 12, pp.165-181.

Lemay, Rodnite H. (1985), «Anthonius Guainerius and Medieval Gynecology», en KIRSHNER, J.-Wemple, S. (eds.), Women of Medieval Word. Essais in Honor of J. H. Mundy, Oxford, Blackwell, pp.317-336.

LiTTRÉ, É. (1839-1863), Oeuvres complètes d'Hippocrate, I-X, París, J.-B. Baillière.

Manuli, P. (1980), «Fisiologia e patologia del femminile negli scritti ippocratici dell'antica ginecologia greca», en GrMeK, M.D. (ed.), Hippocratica, París, CNRS, pp.393-408.

MARTORELLI, R. (2000), «La medicina scolastica tra galenismo e aristotelismo», Studi medievali 41.1, 311-338.

Mazo Karras, R. (2005), Sexuality in Medieval Europe, Nueva York, Routledge.

Montagnana, B. (1497), Consilia Bartholomei Montagnane. Tractatus tres de balneis patauinis. De compositione et dosi medicinarum. Antidotarium eiusdem, Venetiis.

Montero Cartelle, E. (1983), Constanini Liber de coitu, (ed.), Santiago de Compostela, Universidad de Santiago de Compostela.

Montero Cartelle, E. (1987), Ovidio: Arte de amar; Remedios contra el amor; Cosméticos, Madrid, Akal.

Montero Cartelle E. (1988), «Sobre el autor árabe del Liber de coitu y el modo de traducir de Constantino el Africano», Medizinhistorisches Journal 23, 3.4, 213-223.

Montero Cartelle, E. (1990), «Encuentro de culturas en Salerno: Constantino el Africano, traductor», en HAMESSE, J.-FATTORI, M. (eds.), Rencontres de cultures dans la philosophie médiévale. Traductions et Traducteurs de l'antiquité tardive au XIVe siècle, Lovaina-LaNueva - Cassino, Brepols, pp.65-88.

Montero Cartelle, E. (1991a), «Sobre el origen árabe del "Speculum al foderi catalán” y su relación con el Liber minor de coitu salernitano», Anuari de Filologia 14, 71-80.

Montero Cartelle, E. (1991b), El latín erótico, Sevilla, Universidad de Sevilla.

Montero Cartelle, E. (ed.) (1993), Tractatus de sterilitate. Anónimo de Montpellier, Valladolid, Universidad de Valladolid.

Montero Cartelle, E. (1999), «El Lilium Medicinae de Bernardo de Gordon y la literatura medieval sobre la esterilidad», en Aldama, A.M. et alii (eds.), La Filología Latina hoy: Actualización y perspectivas, Madrid, Sociedad de Estudios latinos, pp.709-715.

Montero Cartelle, E. (2008), «El mito de Tiresias: medicina, erotismo y literatura», en SANTAMARÍA, Ma.T. (ed.), La transimisión de la Ciencia desde la Antigüedad al Renacimiento, Cuenca, Ediciones de la Universidad de Castilla-La Mancha, pp.97-116.

Montero Cartelle, E.- Conde Parrado, P.P. (2001), «Sobre nombres y funciones (testes, semen): de la andrología a la ginecología», Medicina nei secoli. Giornale di Storia della medicina 13,2, 373-399. 
RodríGuez Blanco, Ma'.E. (2003), en Tratados Hipocráticos, VIII, Madrid, Gredos.

PAllí, J. (1992), Aristóteles, Investigación de los animales, Madrid, Gredos.

PARÉ, A. (1586), Ambrosi Paraei... de hominis generatione liber, en Casp. Bauhinus, Gynaeciorum siue de mulierum affectibus commentarii Graecorum, Latinorum, Barbarorum, iam olim et nunc recens editorum in tres tomos digesti..., Tomus II, Basileae, per Conradum Waldkirch, ff.404-484

Pellegrini, R. (1968), Sexuología, Madrid, Morata.

RAzES, (1497), Opera omnia Rasis..., Venetiis, per Bonetum Locatellum.

SAliceto, G. De (1490), Summa conseruationis et curationis ... Cyrugia, Venetiis, per Octavium Scotum.

SÁnchez, E. (1994), Aristóteles, Reproducción de los animales, Madrid, Gredos.

SAnZ, L. (1998), Tratados Hipocráticos, IV, Madrid, Gredos.

SARTON, G. (1927-1948), Introduction to the History of Science, Washington-Baltimore.

Savonarola, M. (1502), Practica Ioannis Michaelis Sauonarole..., Venetiis, per Bernardinum Vercellensem.

Schipperges, H. (1964), Die Assimilation der arabischen Medizin durch das lateinische Mittealter, Wiesbaden, Steiner.

Sillamy, N. (1962), Diccionario de Psicología, Barcelona, Plaza y Janes.

Siraisi, N.G. (1987), Avicenna in Renaissance Italy, Princeton, Princeton University Press.

Solomon, M. (1990), Speculum al foder. The Mirror of Coitus, Madison, The Hispanic Seminary of Medieval Studies.

Staden, H. Von (1989), Herophilus. The Art of Medicine in Early Alexandria, Cambridge, Cambridge University Press.

Thomasset, C. (1992), «Aspects de la femme médiévale dans le Lilium medicinae de Bernard de Gordon», en Femmes, Mariages-Lignages, XIIe-XIVe siècles. Mélanges offerts à Georges Duby, Bruselas, De Boeck-Wesmael, pp.361-372.

VÁzQUeZ DE BENITO, C. (ed.), Muhammad ben Abdallah Ibn al-Jatib: Kitab 'amal tabla li-man htbba, (1984), Salamanca, Universidad de Salamanca. 